\title{
Preferencias de adjunción sintáctica de cláusulas de relativo en escolares*
}

\author{
Syntactic attachment preferences of relative clauses in school children
}

\author{
Beatriz Arancibia Gutiérrez ${ }^{1}$, Marcela Bizama Muñoz y Katia Sáez Carrillo ${ }^{3}$ \\ ${ }^{1}$ Universidad Católica de la Santísima Concepción, Facultad de Educación, Departamento de Lenguas, \\ Concepción, Chile. Correo electrónico: barancib@ucsc.cl \\ ${ }^{2}$ Universidad Católica de la Santísima Concepción, Facultad de Educación, Departamento Fundamentos \\ de la Pedagogía, Concepción, Chile. Correo electrónico: mbizama@ucsc.cl \\ ${ }^{3}$ Universidad de Concepción, Facultad de Ciencias Físicas y Matemáticas, Departamento de Estadística, \\ Concepción, Chile. Correo electrónico: ksaez@ucsc.cl
}

Esta investigación estudia las preferencias de adjunción sintáctica de cláusulas de relativo con doble antecedente nominal (El periodista entrevistó a la hija del coronel que tuvo un accidente) en niños de $2^{\circ}, 5^{\circ}$ y $8^{\circ}$ año de enseñanza básica. Se emplearon tres cuestionarios y se compararon las respuestas de los niños con las de 40 estudiantes universitarios de pregrado. Además, en la muestra de escolares se evaluó la memoria de trabajo verbal y la memoria fonológica para examinar posibles relaciones con sus preferencias de adjunción. Los resultados indican que a medida que los niños avanzan en escolaridad, se comportan como los hablantes adultos, es decir, optan preferentemente por la adjunción alta, aunque la animacidad de los sustantivos puede sesgar su interpretación de las oraciones. No hubo correlaciones entre las medidas de memoria y las respuestas a los cuestionarios.

Palabras clave: preferencias de adjunción sintáctica, cláusula de relativo, niños, memoria

This research studies the syntactic attachment preferences of relative clauses with double nominal antecedent (The journalist interviewed the daughter of the colonel who had an accident) in 2nd, 5th and 8th primary school children. Three questionnaires were answered and children's responses were compared with those of 40 undergraduate students. In addition, verbal working memory and phonological memory were measured in the school children to examine possible relationships with their attachment preferences. Results indicate that as children progress through school, they behave like adult speakers, opting for high attachment, although noun animacy can bias their interpretation for the low attachment. There were no correlations between memory measures and the responses to the questionnaires.

Key words: syntactic attachment preferences, relative clause, children, memory

Investigación patrocinada por la Dirección de Investigación de la UCSC (Proyecto DIN 1512). 


\section{INTRODUCCIÓN}

\subsection{La ambigüedad de adjunción sintáctica en las cláusulas de relativo con doble antecedente}

El estudio de las ambigüedades de adjunción sintáctica ha sido ampliamente abordado por la investigación psicolingüística sobre procesamiento sintáctico y comprensión de oraciones. El motivo es que, frente a la opacidad de las operaciones implicadas en el procesamiento sintáctico, las oraciones ambiguas representan, como señalan Carreiras y Meseguer (1999: 165), una verdadera "ventana" que permite observar los mecanismos que usa el procesador para poner a prueba las teorías sobre su funcionamiento. Estas ambigüedades se producen cuando en una oración aparece información que obliga a reanalizar las adjunciones ya procesadas, o bien, cuando el análisis de algún constituyente puede generar dos interpretaciones diferentes pero igualmente plausibles. En el primer caso, hablamos de ambigüedad local o temporal, pues rehacer el primer análisis permite desambiguar la oración. En el segundo caso, en cambio, se trata de una ambigüedad permanente, pues no se cuenta con información que fuerce el análisis en un sentido u otro. Es decir, ambas interpretaciones son posibles, como en El periodista entrevistó a la hija del coronel que tuvo un accidente, en que la relativa puede ser adjuntada a hija o a coronel (Carreiras 1997; Carreiras y Meseguer 1999).

El estudio sobre el procesamiento de oraciones con ambigüedad temporal, conocidas también como "oraciones de vía muerta" o garden path sentences, llevó a las teorías de corte modular a proponer que el procesador recurre a dos estrategias fundamentales de análisis de carácter universal: adjunción alta (mínima) o cierre temprano y adjunción baja o cierre tardío (Frazier y Rayner 1988; Carreiras 1997; Pickering y van Gompel 2006). El principio que subyace a la elección es la economía de recursos de procesamiento: si el procesador se encuentra frente a dos estructuras posibles para la oración, optará por el análisis más simple y que consume menos recursos de memoria, procediendo entonces al cierre temprano. Cuando ambas alternativas de análisis son igualmente complejas, se aplica el principio de cierre tardío, que consiste en adjuntar el nuevo constituyente al sintagma que está siendo procesado en el momento (Carreiras 1992; Mitchell 1994; Carreiras y Meseguer 1999).

Tal sería el caso de las oraciones que incluyen una cláusula de relativo (CR) como parte de un sintagma nominal (SN) complejo del tipo $S N 1+d e+S N 2+C R$, el cual provee dos potenciales sitios de adjunción para dicha cláusula: el SN1 (hija) y el $\mathrm{SN} 2$ (coronel). La teoría sostiene que entonces corresponde aplicar el cierre tardío, que adjunta la cláusula de relativo al SN2. Sin embargo, los resultados de Cuetos y Mitchell (1988) evidenciaron que -a diferencia del inglés- en español la adjunción de estas estructuras respondía más bien al cierre temprano o adjunción alta, lo que ha sido posteriormente confirmado por otros autores (Carreiras 1992; Carreiras y Clifton 1993, 1999; Meseguer et al. 2002; Carreiras et al. 2004; Acuña et al. 2009). Esto ha sido respaldado también en otras lenguas romances, como el gallego (Fraga et al. 2005) y el francés (Zagar et al. 1997; Frenck-Mestre y Pynte 2000), aunque estos últimos han sido rebatidos por Baccino et al. (2000). En portugués e italiano, los datos son controversiales y plantean nuevos problemas (Baccino et al. 2000; Maia et al. 2006). 
La obtención de resultados que iban en contra de la universalidad del cierre tardío llevó a ajustar el modelo con la hipótesis conocida con el nombre de Construal (Gilboy et al. 1995; Frazier y Clifton 1996). Ésta sostiene que el procesamiento de relaciones primarias (como aquella entre el verbo y sus argumentos) se regiría exclusivamente por principios estructurales, mientras que las secundarias (como adjuntos, modificadores y predicados secundarios) serían establecidas por el procesador temático en una fase posterior, siguiendo el principio Construal. Según éste, si un constituyente no puede ser analizado como una relación primaria, debe ser adjuntado al último asignador temático en la oración. Si persiste la ambigüedad, se le interpreta atendiendo características estructurales específicas de la lengua, con cierta permisividad a la injerencia de factores extrasintácticos. Así, la preferencia en español por la adjunción alta de la cláusula de relativo con doble antecedente obedecería al "principio de referencialidad", introducido en el modelo por Frazier (1990), según el cual los modificadores restrictivos (como las cláusulas de relativo especificativas) se adjuntarían a un constituyente que sea referencial, especialmente si son introducidos por un determinante.

Una alternativa a las teorías universales son los modelos basados en la experiencia, principalmente el de Ajuste Lingüístico (Mitchell et al. 1995) y los modelos interactivos conocidos bajo el rótulo de modelos de Satisfacción de Restricciones Múltiples (MacDonald et al. 1994; Trueswell y Tanenhaus 1994; Tanenhaus et al. 2000). El primero sostiene que el procesador sintáctico no se rige sólo por principios estructurales de carácter universal, pues la frecuencia de uso de las estructuras tendría un papel importante en las decisiones de análisis. No obstante, existen datos en lenguas como el inglés, el holandés y el alemán que muestran inconsistencias entre los estudios de corpus y los resultados de algunas investigaciones que no confirman las mismas preferencias de parte de los sujetos (Carreiras y Meseguer 1999; Pickering y van Gompel 2006). Los modelos de satisfacción de restricciones, en tanto, postulan que el sistema recurre a distintas fuentes, no sólo a información estructural, y construye simultáneamente todas las representaciones posibles para la oración, considerando las restricciones impuestas por los distintos tipos de información. Especial importancia se le da a las restricciones que imponen los ítems léxicos de la oración, pues las características de las palabras en sus distintos niveles de análisis (morfológico, fonológico, sintáctico y semántico) pueden generar variadas interpretaciones. Cuando el sistema tiene que elegir alternativas, escoge la interpretación más compatible con la información contextual. Si el contexto es neutro y las alternativas son igualmente consistentes con él, el sistema considera la frecuencia de uso (Duffy et al. 1988; Snedeker y Trueswell 2004).

Una de las propiedades de las palabras que parece tener un efecto en las decisiones de adjunción sintáctica es la animacidad. La evidencia empírica al respecto muestra que cuando SN1 es inanimado y SN2 es animado, se produce un sesgo hacia la adjunción baja en tareas como completar oraciones y responder preguntas. Este efecto se ha observado en holandés (Desmet et al. 2002; Desmet et al. 2006) y en español (Acuña et al. 2009). En portugués europeo, Soares et al. (2010) encontraron lo mismo en una tarea de compleción de oraciones y en una de lectura cronometrada. Por lo tanto, aunque en español las evidencias empíricas respaldan la idea de una preferencia por la adjunción alta de las cláusulas de relativo con doble antecedente, esto puede ser modulado por la animacidad de los sustantivos en competencia, provocando que las preferencias se inclinen por el sitio de adjunción que contenga un sustantivo animado, 
aunque ello pueda favorecer el cierre tardío. Investigaciones con cláusulas de relativo no ambiguas también han encontrado que la animacidad introduce un sesgo en el análisis, que contribuye a modular el efecto de la complejidad sintáctica en las diferencias de procesamiento entre relativas sujeto y relativas objeto (Mak, Vonk y Schriefers 2002; Traxler et al. 2005; Baudiffiera et al. 2011; He y Bauguo 2013).

Como se puede advertir en esta breve revisión, el interés por poner a prueba las distintas teorías sobre la comprensión de oraciones ambiguas ha motivado una gran cantidad de investigaciones en diversas lenguas. No obstante, se advierte aún cierta asimetría entre la investigación llevada a cabo con hablantes adultos y los estudios realizados con niños. En general, el estudio de la comprensión infantil de la sintaxis se ha centrado más en la descripción del curso evolutivo de la adquisición de las estructuras sintácticas, y menos en la investigación en torno a la descripción de los mecanismos subyacentes al procesamiento sintáctico. Sin embargo, al menos en inglés, ésta es un área que en los últimos años ha ido cobrando progresivamente mayor dinamismo, pues su desarrollo no sólo es una contribución al conocimiento sobre cómo procesan los niños la sintaxis, sino sobre el procesamiento sintáctico y la comprensión de oraciones en general (Felser et al. 2003; Snedeker 2009, 2013).

\subsection{Comprensión de oraciones ambiguas en niños: Algunos antecedentes}

¿Cómo comprenden los niños oraciones estructuralmente ambiguas?, ¿siguen las mismas preferencias de análisis que emplean los adultos? Como se señaló anteriormente, el estudio en torno a estas interrogantes es aún incipiente. Parte de las investigaciones disponibles intenta responder si los niños utilizan de manera inmediata (on-line) información del contexto no lingüístico para resolver ambigüedades referenciales que dificultan la comprensión de oraciones. Es decir, buscan aportar nuevas evidencias al debate acerca de la naturaleza del sistema de procesamiento del lenguaje.

Entre estas investigaciones se cuenta la de Trueswell et al. (1999), quienes encontraron que antes de los 8 años de edad a los niños les cuesta tomar decisiones de análisis si éstas se apartan de información estrictamente estructural. También, de acuerdo con este estudio, les cuesta reanalizar las oraciones si la interpretación no es la correcta. En la misma línea, Hurewitz Brown-Schmidt et al. (2000) concluyen que los niños adquieren primero la habilidad para hacer uso de claves lingüísticas, y gradualmente van desarrollando la capacidad para recurrir a información extralingüística o contextual para resolver ambigüedades referenciales. Snedeker y Trueswell (2004) y Snedeker y Yuan (2008), usando las mismas oraciones con niños de alrededor de 5 años de edad, también encuentran un efecto más débil del contexto que el de claves léxicas y prosódicas en su resolución. Sin embargo, el debate no ha sido completamente zanjado. Así lo evidencian Weighall y Altmann (2011) con niños de 6 a 8 años, en el que incluso los niños con una memoria de trabajo más limitada se apoyan en el contexto para comprender oraciones con ambigüedad referencial. Los autores concluyen que los niños parecen recurrir a los mismos mecanismos de análisis que emplean los hablantes adultos, y que su sensibilidad a las claves extralingüísticas evoluciona en la medida en que aumentan sus recursos cognitivos.

En el ámbito de las preferencias de adjunción sintáctica, Felser et al. (2003) comparan las preferencias de niños (edad promedio 6 años 8 meses) y adultos frente 
a oraciones en las que se manipulan la concordancia de número y una preposición (The student photographed the fan of/with the actress who was/where looking happy). Ellos encontraron que los niños eran menos sensibles que los adultos a las claves semánticas proporcionadas por el elemento desambiguador, y se guiaban preferentemente por estrategias estructurales de análisis moduladas por su memoria de trabajo. Así, los niños con mayor capacidad de memoria optaban por la adjunción alta de la cláusula de relativo, en cambio, los niños con una capacidad de memoria más limitada optaban por la adjunción al sintagma nominal más próximo (actress) o cierre tardío, aun cuando ello los condujera a un análisis errado.

Una investigación más reciente es la de Holly y Liversedge (2013), quienes compararon a niños y adultos en dos tareas de lectura cronometrada palabra a palabra. En una de ellas utilizaron oraciones con ambigüedad de adjunción de una frase preposicional (The boy poked the elephant with the long stick/trunk from outside the cage), mientras que en la otra se manipuló una frase adverbial (I think I'll wear the new skirt I bought tomorrow/yesterday). Los resultados mostraron que niños y adultos optaban preferentemente por la adjunción al verbo (adjunción alta). No obstante, los niños -especialmente los de menos edad- tardaban más en detectar el efecto de vía muerta y eran menos eficientes que los adultos al rehacer el análisis, lo que podría relacionarse con una memoria de trabajo más limitada o con mecanismos de control inhibitorio menos desarrollados.

La relación entre comprensión de oraciones y memoria de trabajo, examinada en algunos de los trabajos citados, ha sido objeto de estudio y amplio debate en la investigación psicolingüística sobre comprensión de oraciones. El principio de economía de recursos de procesamiento que subyace a la formulación de las estrategias de cierre temprano y cierre tardío parte del supuesto de que los procesos de análisis de las oraciones consumen recursos de memoria, cuya disponibilidad es limitada. Aunque existe una gran controversia acerca del papel que la memoria de trabajo juega en el procesamiento sintáctico on-line, hay acuerdo en que cumple un papel importante en la comprensión de oraciones en tareas de comprensión off-line.

Al respecto, Caplan y Waters (1999) hablan de procesos interpretativos para referirse a la comprensión inmediata o en línea, y de procesos postinterpretativos para referirse a tareas off-line o fuera de curso en las que se responden preguntas o se emite algún juicio sobre oraciones recientemente leídas o escuchadas. Las investigaciones realizadas con niños utilizan comúnmente pruebas basadas en el recuerdo de dígitos o de palabras para medir la capacidad del retén fonológico (memoria verbal de corto plazo), y pruebas basadas en el test de Daneman y Carpenter (1980) para medir la memoria de trabajo verbal. Así, por ejemplo, Willis y Gathercole (2001) dan cuenta de una relación entre memoria de dígitos y palabras con el desempeño en repetición de oraciones, pero no con comprensión (ambas tareas off-line) en niños de entre 4 y 5 años de edad.

Por su parte, en italiano, Arosio et al. (2011) encontraron que la memoria de dígitos modulaba la comprensión de oraciones con relativa objeto presentadas en modalidad auditiva (self-paced listening) a niños de 9 años de edad promedio, mientras que la memoria de trabajo no se relacionaba con el procesamiento inmediato ni con la comprensión a posteriori de las oraciones. Por último, más recientemente, Boyle et al. (2013), en una investigación con niños de entre 4 y 6 años, han aportado evidencias favorables a la idea de que la comprensión de oraciones complejas se relaciona con la 
medida del buffer episódico (Baddeley 2001) de la memoria de trabajo. En definitiva, al igual que en sujetos adultos, no existe evidencia concluyente respecto del rol de la memoria en el procesamiento de oraciones en niños, por lo que el tema sigue concitando debate e interés por llevar a cabo investigaciones empíricas (Kidd 2013).

\section{El ESTUDiO}

No se han encontrado investigaciones que aborden específicamente las preferencias de adjunción sintáctica de cláusulas de relativo en niños hablantes de español. En este marco, las preguntas que dan origen al estudio son las siguientes: ¿cómo interpretan los niños estas estructuras sintácticas?, ¿siguen las mismas estrategias de análisis que emplean los hablantes adultos? A partir de estas interrogantes se realizó una investigación exploratoria de tipo off-line con una muestra de escolares de enseñanza básica. Para obtener la información, se les presentan tres tareas que implicaban escoger entre la adjunción alta o la adjunción baja de una cláusula de relativo. En el análisis, se examina si se producen cambios en sus preferencias a medida que avanzan en escolaridad (y en edad), y se les compara con un grupo de estudiantes universitarios. Se consideró la animacidad y el género de los sustantivos para observar si producen algún sesgo en las decisiones de adjunción y, además, se examina si hay alguna relación entre estas decisiones y la memoria de trabajo verbal y memoria verbal de corto plazo de los niños de la muestra.

\subsection{Instrumentos}

- Memoria: Se aplicó una versión en español de la Prueba de Memoria de Trabajo Verbal para Niños de Siegel y Ryan (1989), basada a su vez en el test de Daneman y Carpenter (1980), y el subtest de memoria secuencial auditiva de dígitos del Test Illinois de Aptitudes Psicolingüísticas (ITPA). Ambas pruebas son de aplicación individual. La primera se organiza en cinco niveles, cada uno de ellos con tres series de oraciones que van aumentando desde 2 hasta llegar a 5 frases. A las oraciones les falta la última palabra. La tarea del niño consiste en escuchar una oración y completarla con una palabra que le dé sentido. Al término de cada serie, debe repetir las palabras que utilizó respetando el mismo orden. Su administración se suspende cuando el niño falla en todas las series dentro de un mismo nivel. En el ITPA, el niño tiene que repetir series de dígitos cada vez más largas en el mismo orden en que le son presentadas por el administrador de la prueba. También se suspende después de tres intentos fallidos consecutivos. Esta prueba se aplica hasta los 10 años como parte de una batería destinada al diagnóstico clínico y psicopedagógico. Para los fines de la presente investigación, se trabajó con las puntuaciones directas y no con los baremos, por lo que se utilizó incluso con los niños de más edad.

- Preferencias: Se elaboraron tres cuestionarios de lápiz y papel compuestos por 20 oraciones que presentan en el predicado una cláusula de relativo con ambigüedad de adjunción. La cláusula de relativo forma parte de un sintagma complejo de tipo $S N 1+d e+S N 2+C R$. Se utilizaron además 20 oraciones de relleno, de distinto tipo, pero de similar longitud. Cada cuestionario representa una condición distinta, dependiendo de la animacidad y el género de los sustantivos a los que se puede adjuntar la cláusula de relativo: en el cuestionario 1 (C1), se utilizaron dos sustantivos 
animados (A) de distinto género; en el cuestionario 2 (C2), se empleó un sustantivo inanimado (I) y otro animado de distinto género; finalmente, en el cuestionario 3 (C3), ambos sustantivos son animados y del mismo género.

En todos los casos se seleccionaron sustantivos de carácter concreto y que resultaran familiares para los participantes, lo que se decidió considerando la opinión de las profesoras de Lenguaje y Comunicación de cada curso. Ejemplos de los sustantivos empleados son: mamá, hijo, médico, profesor, niño, amigo, policía, ladrón, vendedor, vecino, cantante, mujer, hombre; prueba, juguete, sillón, canción, árbol, mochila, remedio, libro, vestido. Para recoger la información sobre la interpretación de los participantes, en $\mathrm{C} 1$ y C2 se ofrecen dos alternativas para completar la oración, mientras que en $\mathrm{C} 3$ se debe escoger una alternativa para responder a una pregunta sobre el verbo de la relativa. La Tabla 1 muestra ejemplos de los estímulos empleados:

Tabla 1: Ejemplos de las oraciones empleadas en los cuestionarios

\begin{tabular}{|c|c|c|}
\hline Cuestionario & Condición & Ejemplos \\
\hline $\mathrm{C} 1$ & $\begin{array}{c}\mathrm{A}+\mathrm{A} \\
\text { distinto género }\end{array}$ & $\begin{array}{r}\text { El portero se encontró con la hija del vecino que era... } \\
\text { a) amistoso b) amistosa }\end{array}$ \\
\hline $\mathrm{C} 2$ & $\mathrm{I}+\mathrm{A}$ & $\begin{array}{r}\text { El profesor encontró la prueba del niño que estaba... } \\
\text { a) perdida b) suspendido }\end{array}$ \\
\hline C3 & $\begin{array}{c}\text { A }+\mathrm{A} \\
\text { igual género }\end{array}$ & $\begin{array}{l}\text { El director llamó al amigo del niño que faltó a la escuela. } \\
\text { Pregunta: ¿Quién faltó a la escuela? a) el niño b) el amigo }\end{array}$ \\
\hline
\end{tabular}

\subsection{Participantes}

La selección de los participantes se realizó con los siguientes criterios: no estar diagnosticado con Trastornos Específicos del Lenguaje (TEL), no pertenecer al proyecto de integración (presentar desarrollo cognitivo típico) ni presentar dificultades con la lectura, ser hablantes nativos de español y contar con el consentimiento informado del apoderado. Además, para evitar sesgos en la selección de la muestra asociados a factores socioeconómicos, se optó por escolares que no pertenecieran a la población en situación de alta vulnerabilidad social ni al nivel socioeconómico alto. La información requerida para la selección de los participantes se obtuvo de las direcciones técnico-pedagógicas de los establecimientos y de los profesores jefe y de Lenguaje y Comunicación de cada curso. La muestra quedó conformada por niños y niñas de $2^{\circ}, 5^{\circ}$ y $8^{\circ}$ año básico de una escuela de dependencia municipal de la comuna de Concepción y un establecimiento subvencionado de la comuna de Talcahuano. Ambos establecimientos son mixtos. La Tabla 2 señala el total de participantes y su distribución por curso. 
Tabla 2: Muestra de escolares

\begin{tabular}{|c|c|}
\hline Curso $/ \bar{X}$ edad (años, meses) & Muestra \\
\hline $2^{\circ}(7$ años, 3 m. $)$ & 76 \\
\hline $5^{\circ}(10$ años, 4 m. $)$ & 87 \\
\hline $8^{\circ}(13,4$ m. $)$ & 82 \\
\hline TOTAL & 245 \\
\hline
\end{tabular}

Participó también un grupo de 40 estudiantes universitarios de entre $3^{\circ}$ y $5^{\circ}$ año de diferentes carreras. Todos ellos, seleccionados al azar, participaron de manera voluntaria.

\subsection{Procedimientos de recogida de datos}

La administración de todos los instrumentos se realizó dentro de la jornada escolar y estuvo a cargo de la investigadora principal y dos asistentes. Las pruebas de memoria se aplicaron en salas asignadas por la unidad técnica de cada establecimiento. Los cuestionarios fueron aplicados de manera colectiva en cada curso, con la presencia de la profesora a cargo, que cooperaba en mantener la disciplina. Cada niño utilizó el tiempo que requería para leer y responder según su necesidad. Para evitar que la fatiga o la desconcentración llevaran a responder mecánicamente o a dejar en blanco, los cuestionarios fueron respondidos de a uno en distintas semanas del primer semestre del calendario escolar, comenzando por los niños de octavo hasta llegar a los de segundo año. De esta forma también se buscaba controlar los posibles efectos de la repetición de la tarea en las respuestas. A los niños ausentes el día de alguna aplicación, se les citó en otro horario, en una sala aparte, sin interrupción de otras personas.

\section{Resultados}

\subsection{Tratamiento de los datos}

Se utilizó la prueba t de Student (prueba de Wilcoxon) para establecer estadísticamente cuál era el tipo de adjunción preferida en cada grupo participante. Para establecer si había diferencias significativas entre las respuestas de cada curso y el grupo de jóvenes se utilizó la prueba de Mann-Whitney. El examen de la asociación entre preferencias de adjunción y memoria se efectuó mediante el coeficiente de correlación de Spearman. Para las comparaciones entre los cursos se aplicó un análisis de varianza (prueba de Kruskal-Wallis). Se utilizó el test de Shapiro-Wilk para verificar el supuesto distribucional de normalidad, y se trabajó con un nivel de significancia del 0,05 en cada una de las pruebas aplicadas. 


\subsection{Preferencias de adjunción}

El Gráfico 1 presenta los porcentajes de adjunción baja y alta obtenidos de las respuestas de los tres grupos de escolares más el grupo de jóvenes en el primer cuestionario.

Gráfico 1: Resultados cuestionario 1 ( $\mathrm{A}+\mathrm{A}$ distinto género)

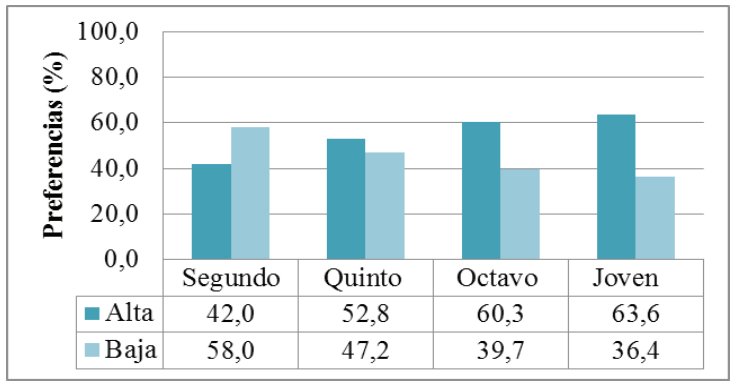

Bajo las barras se muestran los porcentajes correspondientes a los tipos de adjunción que se deducen de las respuestas de cada grupo. Como se puede observar, sólo los niños de segundo presentan una mayor frecuencia de respuestas (58\%) que favorecen la opción por la adjunción baja o cierre tardío de la cláusula de relativo. El análisis de los datos confirmó esa preferencia, que resultó estadísticamente significativa $(\mathrm{p}=0,0105)$. En quinto año básico, la adjunción alta representa un $52,8 \%$ de las respuestas, no obstante, el examen estadístico reveló que esto no alcanzaba a tener un valor significativo. En cambio, en octavo y en el grupo de jóvenes se observó que fue significativa la preferencia por la adjunción alta $(60,3 \%, \mathrm{p}=0,0011$ y $63,6 \%$, $\mathrm{p}=0,0005$, respectivamente). Las comparaciones intergrupales, en tanto, confirmaron que había diferencias entre el comportamiento de los niños de segundo y los otros dos grupos de escolares ( $\mathrm{p}=0,001)$, así como con los jóvenes $(\mathrm{p}<0,0001)$.

En las oraciones de este cuestionario, los sustantivos a los cuales se podía adjuntar la cláusula de relativo eran animados. En C2, en cambio, se construyeron oraciones en las que SN1 era inanimado y SN2, animado. Los resultados se exponen en el siguiente gráfico.

Gráfico 2: Resultados Cuestionario $2(\mathrm{I}+\mathrm{A})$

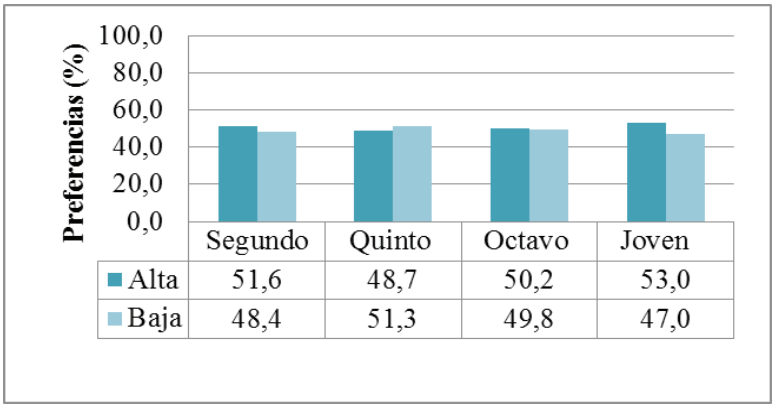


En este caso, se aprecia que los grupos se comportaron de similar manera entre sí. Llama la atención que el mismo patrón se haya observado con independencia del nivel escolar y de la edad. En efecto, el análisis estadístico de los datos no encontró diferencias significativas entre la adjunción alta y baja en ninguno de los niveles escolares. Las comparaciones arrojaron un valor $\mathrm{p}=0,4954$ en $2^{\circ}, 0,5934$ en $5^{\circ}$ y 0,9508 en $8^{\circ}$. Coincidentemente, tampoco fue posible establecer una interpretación preferida por parte de los jóvenes $(\mathrm{p}=0,34)$. Adicionalmente, las comparaciones entre los tres cursos así como entre cada curso con el grupo de jóvenes confirmaron que no había diferencias en el comportamiento de las respuestas $(\mathrm{p}=0,7238)$.

Por lo tanto, los resultados del segundo cuestionario no constituyen evidencia que respalde la hipótesis del cierre temprano como estrategia de análisis de las oraciones ambiguas de tipo $S N 1+d e+S N 2+C R$ en español, pero tampoco favorecen la hipótesis del cierre tardío o adjunción baja. Ahora bien, en todas las oraciones el primer sustantivo era de tipo inanimado. Esto podría estar sesgando el análisis hacia SN2, provocando así un aumento de las preferencias por el cierre tardío que empareja la frecuencia de selección de ambos tipos de adjunción. En el cuestionario siguiente todas las oraciones se construyeron con sustantivos animados, al igual que en $\mathrm{C} 1$, pero del mismo género. Esto podría producir una mayor competencia entre ambos sustantivos por ser el sitio de adjunción de la cláusula de relativo, lo que podría reflejarse en una menor diferencia entre la adjunción alta y baja. Los datos obtenidos fueron los siguientes:

Gráfico 3: Resultados Cuestionario 3 (A + A igual género)

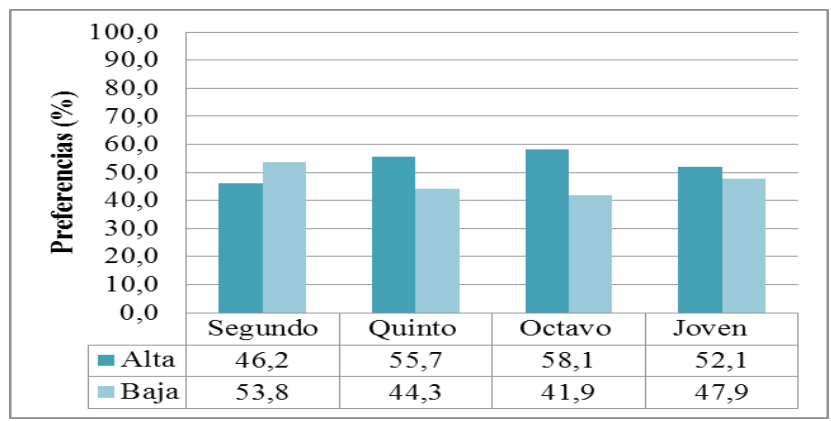

Tal como se observa, en segundo básico la adjunción baja fue más frecuente que la opción por la adjunción alta. No obstante, a diferencia de lo obtenido en C2, el análisis mostró que en este nivel escolar no había respaldo estadístico para establecer que el cierre tardío o adjunción baja fuera la interpretación preferida $(\mathrm{p}=0,0732)$. En cambio, en $5^{\circ}$ y $8^{\circ}$ las preferencias se inclinaron de manera significativa a favor de la adjunción alta de la cláusula de relativo, es decir, al sustantivo que encabeza el sintagma nominal complejo del cual forma parte $(\mathrm{p}=0,0191 \mathrm{y} p=0,0097$, respectivamente). Estos resultados explican que -al igual que en C2- en C3 los niños de segundo se diferencian significativamente de sus pares de $5^{\circ}$ y $8^{\circ}(p=0,0010)$, que se comportan de igual manera entre sí. En el caso de los jóvenes, aunque la 
adjunción alta sobrepasa el $50 \%$, no fue posible con los actuales datos establecer estadísticamente esa preferencia $(\mathrm{p}=0,6368)$, como tampoco diferencias al comparar sus respuestas con las de los niños.

\subsection{Pruebas de memoria}

Los resultados de las pruebas de memoria aplicadas a la muestra de escolares se describen en las Tablas 3 y 4.

Tabla 3: Resultados ITPA

\begin{tabular}{|l|c|c|}
\hline Curso & Media (D.E.) & Mediana (Q1-Q3) \\
\hline Segundo & $9,4(2,4)$ & $9,0(7,0-11,0)$ \\
\hline Quinto & $11,5(3,4)$ & $11,0(9,0-14,0)$ \\
\hline Octavo & $13,9(3,9)$ & $13,0(12,0-16,0)$ \\
\hline
\end{tabular}

Tabla 4: Resultados amplitud de memoria

\begin{tabular}{|l|c|c|}
\hline Curso & Media (D.E.) & Mediana (Q1-Q3) \\
\hline Segundo & $2,33(0,62)$ & $2(2-3)$ \\
\hline Quinto & $2,92(0,77)$ & $3(2-3)$ \\
\hline Octavo & $3,27(0,67)$ & $3(3-4)$ \\
\hline
\end{tabular}

Como era de esperarse, los datos de ambas pruebas evidencian que tanto la capacidad del retén fonológico como de la memoria de trabajo van aumentando a medida que los niños avanzan en escolaridad. En ambas pruebas la comparación entre los cursos arrojó que había diferencias significativas entre los mismos $(\mathrm{p}<0,0001)$.

En cuanto al examen de posibles relaciones entre memoria fonológica y preferencias de adjunción, sólo se encontró que en los niños de $5^{\circ}$ había una correlación positiva baja $\left(r_{s}=0,30\right)$, aunque estadísticamente significativa $(\mathrm{p}=0,005)$, con la preferencia por la adjunción alta manifiesta en $\mathrm{C} 2$. No obstante, como se señaló anteriormente, dicha preferencia no alcanzó significación estadística. Los resultados, por lo tanto, coinciden con investigaciones como las de Willis y Gathercole (2001) y Arosio et al. (2011), que tampoco encuentran una asociación entre memoria fonológica y procesamiento postinterpretativo de oraciones en niños. Por su parte, la memoria de trabajo no correlacionó con las respuestas de ninguno de los cuestionarios en ningún grupo de escolares. 


\section{DisCUSIÓN FINAL}

En este trabajo se buscaba averiguar cómo comprenden los niños las cláusulas de relativo con doble interpretación presentadas como estímulos escritos. Al respecto, la investigación con adultos muestra que en español existe una preferencia por la adjunción alta como estrategia de resolución de este tipo de ambigüedad estructural, lo que ha sido observado en estudios basados en métodos off-line y on-line. Sin embargo, diversas investigaciones dan cuenta de que factores no estructurales, como la animacidad, pueden modular la interpretación de estas oraciones, provocando que las decisiones de adjunción se aparten de lo que se ha descrito como estrategia preferida según la lengua.

Sabemos poco sobre estos fenómenos del lenguaje en la infancia. Las investigaciones sobre comprensión de oraciones ambiguas realizadas con niños se han centrado principalmente en estudiar la resolución de ambigüedades referenciales. En términos globales, los resultados de esas investigaciones muestran que -en general- los niños pequeños utilizan los mismos mecanismos estructurales que los hablantes adultos, pero son menos sensibles a la información aportada por el contexto. A medida que avanzan en edad, sus decisiones de análisis son más flexibles y satisfacen mejor las restricciones contextuales.

No se cuenta, en cambio, con antecedentes sobre comprensión de oraciones con ambigüedad de adjunción de cláusula de relativo. En este contexto, el presente estudio buscaba identificar qué tipo de adjunción establecen preferentemente los niños y si la animacidad y el género de los sustantivos que podrían ser sitios de adjunción intervienen en sus decisiones de interpretación. Se indagó también si sus respuestas se relacionaban con las medidas de su memoria fonológica y su memoria de trabajo.

En su conjunto, los datos muestran una mayor prevalencia de la adjunción alta que de la adjunción baja. Sólo en el primer cuestionario hubo un resultado significativo a favor de la adjunción baja o cierre tardío y correspondió a los niños de segundo año básico. De esta forma, los resultados tienden a respaldar la idea de una preferencia en español por la adjunción alta de las cláusulas de relativo con doble antecedente y son, por lo tanto, coincidentes con los estudios que cuestionan la universalidad del principio de cierre tardío o adjunción baja. Junto con ello, los resultados sugieren que, al parecer, los niños más pequeños tenderían a guiarse más por la recencia o proximidad al establecer las relaciones entre los constituyentes sintácticos. A medida que crecen, sus respuestas revelan que sus preferencias de adjunción van evolucionando hasta ser similares a las de los hablantes adultos.

No obstante, un análisis pormenorizado de la información recogida indica que los factores no sintácticos considerados en el estudio afectaron la selección de la estrategia de interpretación en que se basan las respuestas de los participantes. En este sentido, los resultados confirman la importancia de la animacidad en las decisiones de adjunción sintáctica de las estructuras examinadas. En efecto, en las oraciones I + A, las respuestas se distribuyeron en todos los casos de igual forma entre el cierre tardío y el cierre temprano, lo que mostraría que -al igual que en los hablantes adultos- el empleo de un sustantivo inanimado como candidato para la adjunción alta puede sesgar las preferencias de los niños, provocando un aumento de la opción por el segundo sustantivo. Al respecto, es preciso señalar que los resultados también son coincidentes con Acuña et al. (2009), en el sentido de que la presencia de un nombre 
inanimado frente a uno animado, al parecer, únicamente neutralizaría la preferencia por la estrategia de adjunción alta típica del español.

En relación con el factor género, el análisis de los datos recogidos con el tercer cuestionario (A + A igual género) resulta sugerente. Por una parte, las preferencias de adjunción de los niños son más favorables a la adjunción alta incluso en segundo básico, alcanzando porcentajes estadísticamente significativos en quinto y octavo. Por otra parte, se observa una disminución en la frecuencia de respuestas a favor de la adjunción alta en los jóvenes. Por lo tanto, aunque al parecer sería un factor de menor peso en comparación con la animacidad, el género podría estar relacionado con una mayor competencia entre los dos sustantivos como sitios de adjunción, lo que podría haber incidido en las decisiones de análisis de los niños más pequeños y en los jóvenes. Se trata, por supuesto, de una posibilidad que habría que seguir explorando de manera experimental.

En relación con la memoria, los datos confirman que la memoria fonológica y la memoria de trabajo verbal aumentan con la edad y el nivel escolar de los niños. Sin embargo, no se encontraron correlaciones entre estas variables y las respuestas dadas en los cuestionarios. Una posible explicación es que la autoadministración de cuestionarios de lápiz y papel permite que los sujetos relean las oraciones y respondan con la información a la vista. Por lo tanto, el desempeño no depende de almacenar la información en el retén fonológico y luego recuperarla para poder responder, como ocurriría si la tarea consistiera en escuchar las oraciones. En este sentido, aunque los niños deben leer, se trata de una tarea sencilla, que no tensa los recursos de almacenamiento de información ni la coordinación simultánea de almacenamiento y manejo activo de ella.

Desde este punto de vista, el estudio deja en evidencia la necesidad de investigar las preferencias de adjunción de los niños a través de métodos experimentales basados en tareas on-line, al igual como se ha hecho con adultos. Esto permitiría examinar el procesamiento interpretativo de las oraciones a fin de identificar las estrategias iniciales de análisis que adoptan los niños $\mathrm{y}$, al mismo tiempo, averiguar si éstas son moduladas por las medidas de memoria. Junto con ello, habría que manipular intencionadamente las variables extrasintácticas consideradas en el presente estudio, así como otros factores que podrían intervenir en la comprensión inmediata y a posteriori de las cláusulas de relativo con ambigüedad de adjunción, como la frecuencia léxica, las características semánticas del verbo y la longitud de las oraciones.

\section{OBRAS CITADAS}

Acuña, Juan Carlos, Isabel Fraga, Javier García-Orza y Ana Piñeiro. 2009. “Animacy in the Adjunction of Spanish RCs to Complex NPs". The European Journal of Cognitive Psychology 21: 1137-1165.

Arosio, Fabricio, María Teresa Guasti y Natale Stucchi. 2011. "Disambiguating Information and Memory Resources in Children's Processing of Italian Relative Clauses". Journal of Psycholinguistic Research 40: 137-154.

Baccino, Thierry, Marica De Vincenzi y Remo Job. 2000. "Cross-linguistic Studies of the Late Closure Strategy: French and Italian”. En de Vincenzi, Marica y Vincenzo Lombardo (Eds.), Cross-linguistic Perspectives on Language Processing. Boston: Kluwer Academic Publishers. 89-118. 
Baddeley, Alan. 2001. “Is Working Memory Still Working?”. American Psychologist 56: 849864.

Baudiffiera, Vanessa, David Caplan, Daniel Gaonac'h y David Chesnet. 2011. "The Effect of Noun Animacy on the Processing of Unambiguous Sentences: Evidence from French Relative Clauses". The Quarterly Journal of Experimental Psychology 64.10: 1896-1905.

Boyle, Whitney, Annukka Lindell y Evans Kidd. 2013. "Investigating the Role of Verbal Working Memory in Young Children's Sentence Comprehension”. Language Learning 63.2: 211-242.

Caplan, David y Gloria Waters. 1999. "Verbal Working Memory and Sentence Comprehension". Behavioral and Brain Sciences 22: 77-126.

Carreiras, Manuel. 1992. "Estrategias de análisis sintáctico en el procesamiento de frases: Cierre temprano versus cierre último". Cognitiva 4: 3-27. . 1997. "Diseño funcional y computacional del sistema". En Carreiras, Manuel, Descubriendo y procesando el lenguaje. Madrid: Trotta. 113-183.

y Charles Jr. Clifton. 1993. "Relative Clause Interpretation Preferences in Spanish and English". Language and Speech 36: 353-372.

y Charles Jr. Clifton. 1999. "Another Word on parsing Relative Clauses: Eyetracking Evidence from Spanish and English". Journal of Memory and Language 27.5: 826-833. y Enrique Meseguer. 1999. "Procesamiento de ambigüedades sintácticas". En De Vega, Manuel y Fernando Cuetos (Coords.), Psicolingüistica del español. Madrid: Trotta. 163203.

, Elena Salillas y Horacio Barber. 2004. "Event-related Potentials Elicited during parsing of Ambiguous Relative Clauses in Spanish”. Cognitive Brain Research 20: 98-105.

Cuetos, Fernando y Don Mitchell. 1988. "Cross-linguistic Differences in parsing: Restrictions on the Use of the Late Closure Strategy in Spanish". Cognition 30: 73-105.

Daneman, Meredyth y Patricia Carpenter. 1980. "Individual Differences in Working Memory and Reading". Journal of Verbal Learning and Verbal Behavior 19: 450-466.

Desmet, Timothy, Marc Brysbaert y Constantijn De Baecke. 2002. "The Correspondence between Sentence Production and Corpus Frequencies in Modifier Attachment". Quarterly Journal of Experimental Psychology 55.A: 879-896.

, Constantijn De Baecke, Denis Drieghe, Marc Brysbaert y Wietske Vonk. 2006. "Relative Clause Attachment in Dutch: On-line Comprehension Corresponds to Corpus Frequencies when Lexical Variables are taken into Account". Language and Cognitive Processes 21.4: 453-485.

Duffy, Susan, Robin Morris y Keith Rayner. 1988. "Lexical Ambiguity and Fixation Times in Reading”. Journal of Memory and Language 27: 429-446.

Felser, Claudia, Theodore Marinis y Harald Clahsen. 2003. "Children's Processing of Ambiguous Sentences: A Study of Relative Clauses Attachment”. Language Acquisition 11: 127-163.

Fraga, Isabel, Javier García-Orza y Juan Carlos Acuña. 2005. "La desambiguación de oraciones de relativo en gallego: Nueva evidencia de adjunción alta en lenguas romances". Psicológica 6: 243-260.

Frazier, Lyn y Keith Rayner. 1988. "Parameterizing the Language Processing System: Leftversus Right-branching within and across Languages". En Hawkens, John (Ed.), Explaining Language Universals. Oxford: Blackwell. 247-279.

. 1990. "Parsing modifiers: Special Purpose Routines in the HSPM?". En Balota, David, Giovanni Flores D’Arcais y Keith Rayner (Eds.), Comprehension Processes in Reading. Hills-Dale, N. J.: Lawrence Erlbaum. 303-330.

y Charles Jr. Clifton. 1996. Construal. Cambridge, MA: MIT Press.

Frenck-Mestre, Cheryl y Jöel Pynte. 2000. “«Romancing» Syntactic Ambiguity: Why the French and the Italians don't see Eye to Eye”. En Kennedy, Alan, Ralph Radach, Dieter 
Heller y Jöel Pynte (Eds.), Reading as a Perceptual Process. Oxford: Elsevier. 594-564. Gathercole, Susan y Alan Baddeley. 1993. Working Memory and Language. New York: Psychology Press.

Gilboy, Elizabeth, Josep M. Sopena, Charles Clifton y Lyn Frazier. 1995. “Argument Structure and Association Preferences in Spanish and English Complex NPs". Cognition 54.2: 131-167.

He, Wenguang y Chen Baoguo. 2013. "The Role of Animacy in Chinese Relative Clause Processing". Acta Psychologica 144.1: 145-153.

Holly, Joseph y Simon Liversedge. 2013. “Children's and Adults' On-Line Processing of Syntactically Ambiguous Sentences during Reading”. PLoS ONE 8.1: e54141. doi:10.1371/ journal.pone.0054141

Hurewitz, Felicia, Sara Brown-Schmidt, Kirsten Thorpe, Lila Gleitman y John C. Trueswell. 2000. "One Frog, Two Frog, Red Frog, Blue Frog: Factors Affecting Children's Syntactic Choices in Production and Comprehension". Journal of Psycholinguistic Research 29.6: 597-626.

Kidd, Evan. 2013. "The Role of Verbal Working Memory in Children's Sentence Comprehension: A Critical Review". Topics in Language Disorders 33.3: 208-223.

Maia, Marcus, Eva Fernández, Armanda Costa y Maria Do Carmo Lourenço-Gomes. 2006. "Early and Late Preferences in Relative Clause Attachment in Portuguese and Spanish". Journal of Portuguese Linguistics 5.6: 227-250.

MacDonald, Maryellen, Neal Pearl Mutter y Mark Seidenberg. 1994. "Lexical Nature of Syntactic Ambiguity Resolution". Psychological Review 101.4: 676-703.

Mak, Willem, Wietske Vonk y Herbert Schriefers. 2002. "The Influence of Animacy on Relative Clause Processing". Journal of Memory and Language 47: 50-68.

Meseguer, Enrique, Manuel Carreiras y Charles Clifton. 2002. "Overt Reanalysis Strategies and Eye Movements during the Reading of Mild Garden Path Sentences". Memory and Cognition 30.4: 551-561.

Mitchell, Don. 1994. "Sentence Parsing”. En Gernsbacher, Morton (Ed.), Handbook of Psycholinguistics. San Diego: Academic Press. 375-405.

, Fernando Cuetos, Martin Corley y Marc Brysbaert. 1995. "Exposure-based Models of Human Parsing: Evidence for the Use of Coarse-grained (Nonlexical) Statistical Records". Journal of Psycholinguistic Research 24: 469-488.

Pickering, Martin y Roger van Gompel. 2006. "Sentence Processing". En Gernsbacher, Morton y Matthew Traxler (Eds.), Handbook of Psycholinguistics. San Diego: Academic Press. 455-503.

Siegel, Linda y Ellen Ryan. 1989. “The Development of Working Memory in Normally Achieving and Subtypes of Learning Disabled Children”. Child Development 60.4: 973-980.

Snedeker, Jesse y John Trueswell. 2004. "The Developing Constraints on parsing Decisions: The Role of Lexical-biases and Referential Scenes in Child and Adult Sentence Processing". Cognitive Psychology 49.3: 238-299. y Sylvia Yuan. 2008. "Effects of Prosodic and Lexical Constraints on parsing in Young Children (and Adults)". Journal of Memory and Language 58: 574-608.

2009. "Sentence Processing". En Bavin, Edith (Ed.), The Cambridge Handbook of Child Language. Cambridge: Cambridge University Press. 321-337.

2013. "Children's Sentence Processing". En van Gompel, Roger P. (Ed.), Sentence Processing. Current Issues in the Psychology of Language. New York: Psychology Press. 189-220.

Soares, Ana Paula, Isabel Fraga, Monserrat Comesaña y Ana Piñeiro. 2010. "El papel de la animacidad en la resolución de ambigüedades sintácticas en portugués europeo: Evidencia en tareas de producción y comprensión". Psicothema 22.4: 691-696.

Tanenhaus, Michael, Michael Spyvey-Knowlton y Joy Hanna. 2000. "Modeling Thematic and 
Discourse Context Effects on Syntactic Ambiguity Resolution within a Multiple Constraints Framework: Implications for the Architecture of the Language Processing System". En Pickering, Martin, Charles Clifton y Matthew Crocker (Eds.), Architecture and Mechanism of the Language Processing System. Cambridge: Cambridge University Press. 90-118.

Traxler, Matthew, Rihana Williams, Shelley Blozis y Robin Morris. 2005. "Working Memory, Animacy, and Verb Class in the Processing of Relative Clauses". Journal of Memory and Language 53: 204-224.

Trueswell, John C. y Michael Tanenhaus. 1994. "Toward a Lexicalist Framework for Constraint-based Syntactic Ambiguity Resolution". En Clifton, Charles, Lyn Frazier y Keith Rayner (Eds.), Perspectives on Sentence Processing. New York: Lawrence Erlbaum Association. 155-179.

, Irina Sekerina, Nicole Hill y Marian Logrip. 1999. "The Kindergarten-path Effect: Studying On-line Sentence Processing in Young Children". Cognition 73: 89-134.

Weighall, Anna y Gerry Altmann. 2011. "The Role of Working Memory and Contextual Constraints in Children's Processing of Relative Clauses". Journal of Child Language 38.3: 579-605.

Willis, Catherine y Susan Gathercole. 2001. "Phonological Short-term Memory Contributions to Sentences Processing in Young Children". Memory 9.4/5/6: 349-363.

Zagar, Daniel, Jöel Pynte y Sylvie Rativeau. 1997. "Evidence for Early-closure Attachment on First-pass Reading Times in French". The Quarterly Journal of Experimental Psychology 50.2: 421-38. 